\title{
Intoxicação crônica por Pteridium arachnoideum em bovinos no nordeste do estado do Rio Grande do Sul, Brasil*
}

\section{Chronic poisoning by bracken fern (Pteridium arachnoideum) in cattle in the northeast region of the state of Rio Grande do Sul, Brazil}

\author{
Gilson Antonio Pessoa, ${ }^{* *}$ Jerbeson Hoffman da Silva, ${ }^{* * *}$ Vanessa de Lima, Barbara Guzzon, ${ }^{* * *}$ Alana Mainieri, ${ }^{* * *}$ \\ Gustavo Sogari, ${ }^{* * *}$ Milana Paese, ${ }^{* * *}$ Jonatan Molling Marian, ${ }^{* * *}$ Willian Lanius ${ }^{* * *}$
}

\begin{abstract}
Resumo
Pteridium spp., popularmente conhecida como samambaia, comumente encontrada em solos ácidos, arenosos e de baixa fertilidade. A intoxicação ocorre quando os animais passam por privação alimentar ou ingestão acidental. O objetivo deste estudo é relatar a ocorrência de dois casos de intoxicação crônica por samambaia em bovinos na região nordeste do estado do Rio Grande do Sul, Brasil. Ambos os casos cursaram com sinais clínicos de disfagia, halitose, salivação e presença de tumores na orofaringe. A baixa oferta e qualidade de forragem, superlotação das áreas de pasto, manejo inadequado das pastagens, a presença de Pteridium arachnoideum, os sinais clínicos em conjunto com os achados de necropsia e o exame histopatológico confirmaram o diagnóstico de intoxicação crônica.
\end{abstract}

Palavras chave: Pteridium arachnoideum, plantas tóxicas, Bovino.

\begin{abstract}
Pteridium spp., commonly known as fern, is commonly found in acidic, sandy and low fertility soils. Poisoning occurs when animals go through food deprivation or accidental ingestion. The objective of this study is to report the occurrence of two cases of chronic intoxication in cattle in the northeast region of the state of Rio Grande do Sul, Brazil. Both cases had clinical signs of dysphagia, halitosis, salivation and the presence of tumors in the oropharynx. Low supply and quality of forage, overcrowding of pasture areas, inadequate pasture management, presence of Pteridium arachnoideum, clinical signs, necropsy and histopathological of lesions confirmed the diagnosis of chronic intoxication.
\end{abstract}

Keywords: Pteridium arachnoideum, poisonous plants, Cattle.

\section{Introdução}

A área pastoril do Rio Grande do Sul é composta por quase $70 \%$ de campo nativo, onde é possível encontrar regiões com pastagens grosseiras e espécies indesejáveis. Nestas áreas, é comum a prática das queimadas como forma de controle deste tipo de vegetação (Ribas e Massuquetti, 2004). A região da serra gaúcha possui predominância de solos ácidos e este manejo de solo costuma agravar ainda mais o problema, pois aumenta a acidez dos solos e interfere na disponibilidade de nutrientes.

A planta Pteridium spp. popularmente conhecida como samambaia, caracteriza-se por ser perene, rizomatosa, herbácea e ramificada, medindo entre $50 \mathrm{~cm}$ e $180 \mathrm{~cm}$. É comumente encontrada em solos ácidos e tóxica em toda sua extensão (Nicholson, 2011; Nogueira e Andrade, 2011; Furlan et al, 2014). O rizoma, caule que cresce horizontalmente, é a parte mais tóxica e, nas partes aéreas a toxidez é maior na brotação pela maior concentração de ptaquilósidio (Bischoff e Smith, 2011; Nicholson, 2011; Nogueira e Andrade, 2011; Plessers et al., 2013; Souto et al. 2006).

A samambaia não é atrativa para o consumo, exceto em períodos de pouca disponibilidade de alimento. A ocorrência da intoxicação e os sinais clínicos observados, dependem da quantidade ingerida da planta e do tempo de exposição. Sabe-se que os rizomas expostos após a aragem e os brotos jovens são mais palatáveis e apresentam maior toxicidade. A contaminação do feno com samambaia também pode causar intoxicação, pois mesmo seca ainda possui toxicidade (Andrews et al., 2004).

O glicosídeo norsesquiterpeno ptaquilosídeo é o princípio contido na planta que apresenta contribuição significativa na atividade carcinogênica e mutagênica (Castillo et al., 1997; Marçal et al., 2001). Bischoff e Smith (2011) relatam que $42 \%$ dos casos de intoxicações por $P$. aquilinum no rebanho brasileiro apresentam neoplasias no trato gastrointestinal.

\footnotetext{
${ }^{*}$ Recebido em 27 de março de 2018 e aceito em 3 de maio de 2019.

**Universidade de Caxias do Sul

${ }^{* * *}$ Professor do Departamento de Clínica de Grandes Animais da Universidade Federal de Santa Maria (UFSM).
} 
A ação radiomimética é relacionada a quadros clinico patológicos de carcinoma epidermoide, diátese hemorrágica e hematúria enzoótica bovina. Em bovinos a intoxicação pode-se apresentar como superaguda, aguda, subaguda ou crônica (Riet- Correa e Medeiros, 2001).

A forma aguda é evidenciada quando ocorre a ingestão diária superior a $10 \mathrm{~g} / \mathrm{kg}$ de peso vivo durante três semanas a poucos meses. Já a forma crônica, o animal ingere quantidade menor que a relatada anteriormente, porém por período mais longo de meses a anos e apresenta hematúria enzoótica e formação de carcinomas epidermoides no trato digestivo superior (Gabriel et al. 2009; Nogueira e Andrade, 2011; Souza e Graça, 1993).

Os sinais clínicos variam de acordo com a quantidade de planta ingerida e tempo de exposição. Em animais que apresentam carcinomas epidermóides, os sinais variam de acordo com a localização dos tumores. Por exemplo, quando estes estão presentes na região orofaríngea, o animal apresenta disfagia, regurgitação, ptialismo, halitose, tosse e dispneia. Quando presentes no esôfago ou cárdia, podem desenvolver os sinais anteriormente citados, além de timpanismo ruminal intermitente devido a falha na eructação (Nogueira e Andrade, 2011; Souza e Graça, 1993).

A ingestão de samambaia pode causar danos a medula óssea, prejudica a formação de elementos figurados do sangue, resulta em grave leucopenia e trombocitopenia, semelhante aos danos causados pelo elemento rádio. O animal manifesta quadro hemorrágico generalizado, caracterizado por hemorragias nasal, oral ou vaginal. As mucosas podem apresentar petéquias, sufusões e há relatos de hematidrose que evolui para quadro de hipertermia $\left(41^{\circ} \mathrm{C}\right.$ a $42^{\circ} \mathrm{C}$ ) (Nogueira e Andrade, 2011).

Existem evidencias de que o efeito radiomimético de $P$. aquilinum também se manifeste em seres humanos. A planta é consumida em algumas partes do mundo, notadamente no Japão, no nordeste dos Estados Unidos, Canadá, China e Sibéria. Além disso, os riscos à população humana não se restringem apenas ao consumo direto da planta, mas também à ingestão do princípio carcinogênico através de leite e laticínios (França et al., 2002).

O objetivo deste relato visa contribuir com informações sobre a intoxicação crônica pelo consumo de samambaia em bovinos utilizados na produção de leite para subsistência.

\section{Material e métodos}

Os casos relatados neste artigo são provenientes de pequenas propriedades rurais localizadas na região da serra gaúcha nos municípios de Caxias do Sul e São Marcos, atendidos na Clínica médica de Ruminantes da Universidade de Caxias do Sul.

\section{Caso 1}

Em setembro de 2016, foi atendido uma fêmea bovina, sem raça definida, com cerca de 7 anos de idade e cerca de $350 \mathrm{~kg}$ de peso corpóreo. O animal encontrava-se em período de puerpério (40 dias pós-parto). O mesmo era mantido em sistema extensivo, em pastagem de campo nativo, que no inverno é composto por espécies como o capim caninha (Andropogon lateralis), da barba-de-bode (Aristida sp.), do gravatá (Eryngium sp), além de leguminosas nativas, com destaque para o pega-pega (Desmodium incanum) (Carvalho, Maraschin e Nabinger, 1998).
Os animais da propriedade não eram submetidos à manejos de vacinações ou controle antiparasitário.

O proprietário relatou que o animal apresentava emagrecimento progressivo, diarreia, disfagia, sialorreia, refluxo e halitose. Os sinais clínicos tiveram início há cerca de um ano e nenhum tratamento foi instituído. Ao realizar exame físico o animal de acordo ao descrito Radostits (2006), observou-se baixo escore de condição corporal 2 (escala de 1 a 5), anorexia, desidratação (superior a 8\%), mucosas hipocoradas a ictéricas, taquicardia, taquipneia, hipomotilidade ruminal, normotermia e timpanismo discreto. Ao exame da cavidade orofaríngea, foi possível visualizar a presença de tumoraçõeshalitose e estenose do lúmen esofágico (Figura 1).

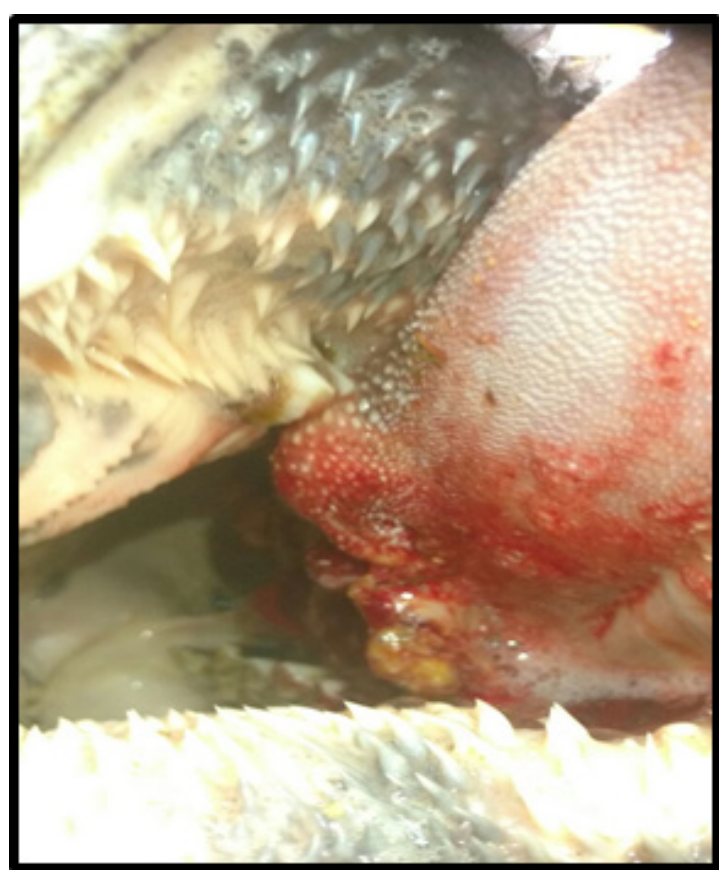

Figura 1: Bovino fêmea com 7 anos de idade com tumorações na base da língua.

No hemograma observou-se anemia normocítica normocrônica e hiperfibrinogênemia. Os valores plaquetários não apresentaram alterações. O leucograma apresentou leucometria global dentro da normalidade e na leucometria especifica, linfopenia.

Já a presença de leucócitos dentro dos parâmetros pode ser mascarada pela desidratação (valores relativos vs. absolutos). Ao exame bioquímico, o animal apresentou hipocalcemia (cálcio sérico total), diminuição discreta dos níveis séricos de creatinina e elevação de proteínas; os quais podem ser causados pelo quadro de anorexia e desidratação apresentados pelo animal(Radostits, 2006).

Considerando os dados obtidos através da inspeção, anamnese, exame clinico completo e exames complementares; aliados ao fato de os animais eram mantidos em um campo com escassez de forragens e com a presença de grande quantidade da referida planta tóxica, chegou-se à conclusão que o diagnóstico mais provável era de intoxicação crônica por consumo de samambaia.

O prognóstico foi desfavorável, porém instituiu-se tratamento sintomático utilizando-se diclofenaco de sódio $\left(1 \mathrm{mg} / \mathrm{kg}^{-1}\right)$ via 
intramuscular (IM) durante 3 dias e oxitetraciclina L.A. $(20$ mg/kg$\left.{ }^{1}\right)$ via IM a cada $48 \mathrm{~h}$ em três aplicações, além da administração de solução de cálcio glicosado IV (frasco com volume de 500 $\mathrm{mL}$ ) e hidratação via oral, através de sondagem orogástrica e administração de uma solução energética e hidroeletrolítica balanceada, com volume total de 30 litros em uma única aplicação.

Devido ao fato de o proprietário não possuir outra fêmea em lactação no momento para alimentação da bezerra foi recomendado manter o animal até ser possível realizar 0 desmame e posteriormente realizar eutanásia.

\section{Caso 2}

No dia 20 de março de 2017, foi atendido na Clínica de Ruminantes da Universidade de Caxias do Sul, uma fêmea bovina da raça brangus, de nove anos de idade, com peso aproximado de $350 \mathrm{~kg}$. O exame clínico foi realizado segundo os parâmetros descritos por Radostitis (2006). O animal apresentava hiporexia, emagrecimento progressivo, escore corporal 2 (escala de 1-5), disfagia, desidratação de $8 \%$, mucosas hipocoradas e diarreia que perdurava a cerca de três meses.

Foram ofertados pasto e feno ao animal, o qual apresentou regurgitação do conteúdo (Figura 2). Diante do quadro clínico, o médico veterinário responsável optou pela passagem de sonda oroesofágica, não obtendo sucesso devido a presença de estenose esofágica.

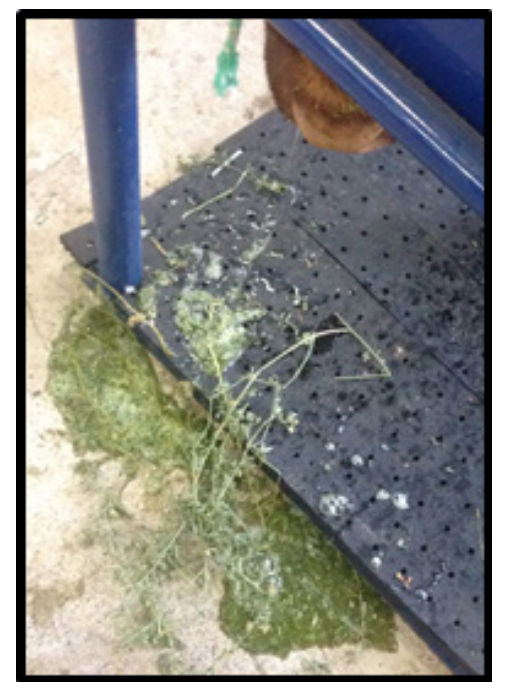

Figura 2: Regurgitação de após fornecimento de pasto e feno a fêmea bovina de 9 anos de idade.

Devido a baixa condição corporal, realizou-se exame coproparasitológico de fezes (OPG), pela técnica Gordon \& Whitlock segundo Ueno \& Gonçalves (1994), o qual revelou a presença de oocistos de Eimeria (1200), ovos de Monieza (400) e estrongilideos (1200). No hemograma, observou-se anemia normocítica normocrônica, e, no leucograma, leucocitose por neutrofilia. Nos exames bioquímicos constatou-se hipocalcemia (6,2 $\mathrm{mg} / \mathrm{dL})$, e aumento nos níveis séricos de gama glutamil transferase (GGT) (52,0 U/I) (Radostits,2006). Os do hemograma e bioquímico consiste com os relatados por Falbo et al. (2005).

O tratamento instituído foi baseado no uso de ceftiofur (1mg/ $\mathrm{kg}$ ) IM a cada 24 horas durante 3 dias, Flunixina meglumina $(2.2 \mathrm{mg} / \mathrm{kg})$ IM a cada 24 horas por 3 dias e fluidoterapia utilizouse 10 litros ringer lactato, $250 \mathrm{ml}$ de glicose a $5 \%$ e $500 \mathrm{ml}$ de multivitamínico na velocidade de $15 \mathrm{ml} / \mathrm{kg} / \mathrm{h}$.

Por não apresentar melhoras no quadro clínico optou-se pela realização de laparotomia exploratória, seguida de rumenotomia, segundo a técnica descrita por Hendrickson e Baird (2013). Com isso, detectou-se a presença de tumorações na porção final do esôfago, os quais eram responsáveis pelo quadro de obstrução e consequente regurgitação.

Devido à ausência de melhora e prognostico desfavorável, optou-se pela realização de eutanásia. Na necropsia, observouse congestão hepática e hepatomegalia, além da presença de massas tumorais na porção final do esôfago e região do cárdia (Figura 3), os quais foram classificados como carcinoma de células escamosas (CCE) no exame histopatológico. Tais achados corroboram para a suspeita inicial de intoxicação crônica por samambaia. Ainda no exame histopatológico, os fragmentos de fígado analisados apresentaram áreas de necrose acompanhadas de infiltrados de neutrófilos, classificado no exame morfológico como hepatite necrótica supurativa multifocal.

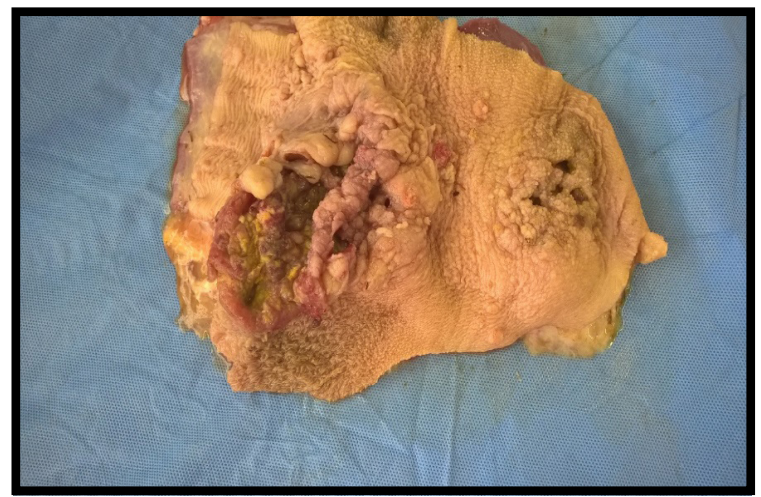

Figura 3: Presença de massa tumoral no cárdia de bovino fêmea, 9 anos de idade.

\section{Discussão}

Os dois bovinos eram provenientes de propriedades que apresentavam grande quantidade de samambaia nas áreas de pastoreio e manejo inadequado do solo em uma região declivosa. Segundo os criadores haviam ocorridos casos com sinais clínicos semelhantes em anos anteriores e em propriedades vizinhas. No entanto, os criadores desconheciam que a planta possui efeitos tóxicos aos animais.

Em ambos os casos, os achados clínicos são condizentes com o que está descrito na literatura (Souto et al., 2006; Gabriel et al. 2009; Souza e Graça, 1993; Nogueira e Andrade, 2011; Plessers et al., 2013). O diagnóstico de intoxicação crônica por Pteridium arachnoideum foi baseado nos aspectos clínicos, 
hematológicos e patológicos, além da presença da planta tóxica. Os dois animais apresentaram CCEs no trato alimentar superior (TAS), apatia, hiporexia, disfagia e palidez das mucosas, o que caracteriza a forma crônica da enfermidade (Souto et al., 2006).

A escassez de forragens no local em que estes animais eram manejados justifica o consumo da planta, visto que normalmente os bovinos são relutantes em ingeri-la, em decorrência de sua baixa palatabilidade. Caso o consumo ocorra, alguns bovinos podem desenvolver "vício" pela planta e passar a ingerir a mesma com maior frequência (Anjos et al.,2009; Plessers et al., 2013).

Quanto aos dados hematológicos, ocorre depressão da medula óssea induzida pela samambaia, a qual resulta em leucopenia grave (particularmente granulocitopenia) principalmente na forma aguda. Na forma crônica, as alterações hematológicas ocorrem principalmente em decorrência do quadro de caquexia induzido pela intoxicação (Gabriel et al., 2009; Nicholson, 2011; Bischoff e Smith, 2011; Riet-Correa e Medeiros, 2001).

No primeiro caso foi observado quadro de anemia normocítica normocrômica, com linfopenia. Já no segundo caso, o animal também apresentou anemia normocítica normocrômica e leucocitose por neutrofilia, justificado pela necrose hepática supurativa multifocal (Radostits, 2006). A principal alteração hematológica observada nas formas crônicas da intoxicação é anemia arregenerativa, não sendo visto nos casos aqui descritos, em alguns casos, podem ocorrer alterações no leucograma (Riet- Correa e Medeiros, 2001).

A forma crônica da intoxicação é mais comum em animais acima dos 5 anos de idade, pois para que a mesma ocorra, é necessário que o indivíduo seja exposto a planta por longo período (RietCorrea e Medeiros, 2001). Ambos os animais relatados neste caso apresentavam idade superior a 5 anos.

A samambaia ativa os oncogênes das células do trato digestório superior e urinário (Bischoff e Smith, 2011). Ambas as formas são responsáveis por grandes perdas econômicas em regiões endêmicas, porém a prevalência dessas formas varia de região para região (Tokarnia et al., 1969; Riet-Correa e Medeiros, 2001).

A ocorrência de CCE no trato alimentar é a forma de manifestação crônica da enfermidade. Os CCEs ocorrem geralmente na base da língua, esôfago, cárdia e rúmen. Como consequência da interferência mecânica no processo de deglutição de ruminação, os animais acometidos geralmente apresentam um quadro de emagrecimento crônico. Os sinais clínicos mais comuns nesta forma da enfermidade, podem incluir: apatia, anorexia, tosse, regurgitação, timpanismo, diarreia, melena, hematoquezia, perda de peso progressiva e morte (Riet-Correa e Medeiros, 2001; Bischoff e Smith, 2011; Radostits, 2006). Os achados em ambos os casos estão em concordância com o que é relatado na literatura.

Os tumores localizados no trato digestivos superior são macroscopicamente semelhantes aos relatados por Masuda et al. (2011) e justificam alguns sinais clínicos observados. Os achados nos exames histopatológicos, determinaram que, em ambos os animais, as tumorações eram compatíveis com CCE, fato comum na enfermidade em questão (Radostits, 2006).
Quanto ao segundo caso, além da presença de carcinomas de células escamosas do trato alimentar, característico da forma crônica da enfermidade, também se observou hepatite necrótica supurativa multifocal aleatória moderada. Este padrão de lesão pode ser observado na forma aguda da intoxicação por samambaia devido a septicemia (Anjos et al.,2009). Contudo, essa sobreposição das formas aguda e crônica da intoxicação por samambaia é incomum e não relatada na literatura.

O tratamento indicado nos casos de intoxicação por samambaia é apenas sintomático e o prognóstico é desfavorável (Bischoff e Smith 2011; Riet- Correa e Medeiros, 2001; Radostits, 2006; Galvão et al., 2012). A prevenção pode ser realizada com a retirada de animais de pastagens muito contaminadas, ou a rotação entre pastagens contaminadas e não contaminadas. $\mathrm{O}$ corte estratégico da planta durante o inverno, assim como o uso de herbicidas, também são formas de controle. É aconselhável realizar a inspeção das pastagens antes da transferência do rebanho, prevenção com o oferecimento de alimentação adequada, assim como um sistema de informações referentes as espécies de plantas tóxicas da região (Rissi et al., 2007; Riet-Correa e Medeiros, 2001).

Conforme Rissi et al. (2007), a intoxicação por samambaia pode ter sido subdiagnosticada, pois os bovinos afetados geralmente são provenientes de pequenas propriedades, onde os produtores são desprovidos dos meios necessários para o conhecimento da condição e apoio técnico para diagnóstico. Os tumores do trato digestivo foram descritos como a forma mais comum na intoxicação por $P$. aquilinum em Santa Catarina, onde é considerada a principal causa de morte relacionada ao consumo da planta (Gava, 1993).

O controle das intoxicações por ptaquilosídeos são importantes não somente em bovinos, mas também é de extrema importância na saúde pública. Os riscos à população humana não se restringem apenas ao consumo direto da planta e a ingestão de leite e derivados, mas também através da ingestão de carne de animais intoxicados por plantas Shahin et al. (1999) demonstraram que o leite de vacas que consomem Pteridium spp. contém quantidades significativas de ptaquilosídeo e isto representa um risco para saúde pública, em virtude de suas propriedades cancerígenas. Experimentalmente já se observou que animais que ingeriram leite de vacas que consumiam $P$. aquilinum apresentaram alterações deletérias na atividade da medula óssea (Evans et al., 1982).

\section{Conclusão}

A intoxicação por Pteridium spp. gera importantes percas econômicas na bovinocultura nacional. O manejo incorreto dos solos e a utilização das queimadas são fatores predisponentes para o surgimento da planta. Embora, a literatura científica sobre o assunto seja vasta, muitos criadores ainda desconhecem os efeitos tóxicos da planta aos bovinos.

A ingestão ocorre em momentos de baixa oferta de forragens e altas taxas de lotação, fatos estes que foram observados nos criatórios que encaminharam os bovinos para atendimento. Em ambos os casos relatados, o prognóstico foi desfavorável e a condição, irreversível. Portanto, reforça-se a importância das práticas corretas de manejo como profilaxia. 


\section{Referências}

ANDREWS, A.H.; BLOWEY, R.W.; BOYD, H.; EDDY, G.E. Bovine Medicine: Diseases and Husbandry of Cattle. 2. ed. Oxford: Blackwell Science, 2004.

ANJOS, B.L.; IRIGOYEN, L.F.; PIAZER, J.V.M.; BRUM, J.S.; FIGHERA, R.A.; BARROS, C.S.L. Intoxicação experimental aguda por samambaia (Pteridium aquilinum) em bovinos. Pesquisa Veterinária Brasileira. V. 29, 753-766, set. 2009.

BISCHOFF, K.; SMITH, M. C. Toxic Plants of the Northeastern United States. Veterinary Clinics of North America: Food Animal Practice, v. 27, n. 2, p.459-480, jul. 2011.

CARVALHO, P.C.F.; MARASCHIN, G.E.; NABINGER, C. Potencial produtivo do campo nativo do Rio Grande do Sul. In: PATIÑO, H.O. (Ed.). SUPLEMENTAÇÃO DE RUMINANTES EM PASTEJO, 1, Anais, Porto Alegre-RS. 1998.

CASTILLO, U. F.; WILKINS, A.L.; LAUREN, D.R.; SMITH, B.L.; TOWERS N. R.; ALONSO-AMELOT, M.E.; RAMON, J.E. Isoptaquiloside and caudatoside, illudane-type sesquiterpene glucosides from Pteridium aquilinum var. caudatum. Phytochemistry, [s.I.], v. 44, n. 5, p.901-906, mar. 1997. Elsevier BV. http://dx.doi.org/10.1016/s00319422(96)00593-6.

EVANS, I.A.; JONES, R.S.; MAINWARING - BURTON, R. Passage of bracken fern toxicity into milk. Nature, v. 237, p.107108, 1972.

FALBO, Margarete Kimie et al. Alterações hematológicas, bioquímicas, urinárias e histopatológicas na intoxicação natural em bovinos pela samambaia Pteridium aquilnum (L.) Kühn. Semina: Ciências Agrárias, [s.I.], v. 26, n. 4, p.547-558, 30 jun. 2005. Universidade Estadual de Londrina. http://dx.doi. org/10.5433/1679-0359.2005v26n4p547.

FRANCA, T. N.; TOKARNIA, C. H.; PEIXOTO, P. V. Enfermidades determinadas pelo princípio radiomimético de Pteridium aquilinum (Polypodiaceae). Pesq. Vet. Bras., Rio de Janeiro, v. 22, n. 3, p. 85-96, Sept. 2002.

FURLAN, F.H.; MENDES, E.R.S.; DUCATTI, K. R.; MARCON, G. C.; DOMBROSKY, T.; AMORIM, T. M.; RIET-CORREA, F. Intoxicação aguda por Pteridium arachnoideum e Pteridium caudatum em bovinos e distribuição das plantas em Mato Grosso. Pesq. Vet. Bras., Rio de Janeiro, v. 34, n. 4, p. 343-348, Apr. 2014. Available from <http://www.scielo.br/scielo.php?script=sci_ arttext\&pid=S0100736X2014000400008\&Ing=en\&nrm=iso>. Access on 01 Aug. 2018. http://dx.doi.org/10.1590/S0100736X2014000400008.

GAVA, A. Intoxicação por Pteridium aquilinum, In: Riet-Correa F., Méndez M.C. \& Schild A.L. (ed.), Intoxicações por Plantas e Micotoxicoses em Animais Domésticos. Hemisfério Sul do Brasil, Pelotas. p.247-258. 340p. 1993.

GABRIEL, A.L.; KOMMERS, G.D.; MASUDA, E.K; FIGHERA, R.A.; PIAZER, J.V.M.; BARROS, C.S.L.; MARTINS, T.B.; ROSA, F.B. Aspectos clínico-hematológicos e lesões vesicais na intoxicação crônica espontânea por Pteridium aquilinum em bovinos. Pesq. Vet. Bras. 29 (7):515-525, julho 2009

GALVÃO, Alexandre et al. Sobrevivência/viabilidade de bovinos com Hematúria Enzoótica após transferência para região livre de Pteridium arachnoideum. Pesquisa Veterinária Brasileira, [s.I.], v. 32, n. 9, p.887-902, set. 2012. FapUNIFESP (SciELO). http:// dx.doi.org/10.1590/s0100-736x2012000900013.
HENDRICKSON, D.A.; BAIRD, A.N. Turner and Mcllwraith's techniques in large animal surgery, 4th ed. 331p, 2013.

MARÇAL, W.S.; GASTE, L.; REICHERT NETTO, N.C.; MONTEIRO, F.A. Ocorrência de intoxicação aguda em bovinos pela samambaia (Pteridium aquilinum , L. Kuhn) no norte do Paraná-Brasil. Semina: Ci. Agrárias, Londrina-pr, v. 22, n. 2, p.139-144, dez. 2001.

MASUDA, E.K.; KOMMERS, G.D.; MARTINS, T.B.; BARROS, C.S.L.; PIAZER, J.V.M. Morphological Factors as Indicators of Malignancy of Squamous Cell Carcinomas in Cattle Exposed Naturally to Bracken Fern (Pteridium aquilinum). Journal of Comparative Pathology, v. 144, n. 1, p.48-54, jan. 2011.

NICHOLSON, S. S. Southeastern Plants Toxic to Ruminants. Veterinary Clinics Of North America: Food Animal Practice, v. 27, n. 2, p.447-458, jul. 2011.

NOGUEIRA, R.M.B; ANDRADE, S.F. Manual de Toxicologia Veterinária. São Paulo - SP: Roca, 2011.

PLESSERS, E.; PARDON, B.; DEPREZ, P., DE BACKER P., CROUBLES S. Acute hemorrhagic syndrome by bracken poisoning in cattle in Belgium. Vlaams Diergeneeskundig Tijdschrift, 82: 31-37, (2013).

RADOSTITS, O.M.; GAY, C.C.; HINCHCLIFF, K.W. VETERINARY MEDICINE: A textbook of the diseases of cattle, horses, sheep, pigs and goats. 10. ed. Edinburgh: Elsevier, 2006.

RIBAS, R.J.; MASSUQUETTI, A. A PECUÁRIA DE CORTE GAÚCHA: UMAANÁLISE DOS PRINCIPAIS SISTEMAS DE PRODUÇÃO. UFRGS, Porto Alegre, p.1-31, jul. 2004.

RIET-CORREA, F.; MEDEIROS, R.M.T. 2001. Intoxicações por plantas em ruminantes no Brasil e no Uruguai: importância econômica, controle e riscos para a saúde pública. Pesq. Vet. Bras. 21:38-42.

RISSI D.R.; RECH, R.R.; PIEREZAN, F.; GABRIEL, A.T.; TROST, M.E.; BRUM, J.S.; KOMMERS, G.D.; BARROS, C.S.L. Intoxicações por plantas e micotoxinas associadas a plantas em bovinos no Rio Grande do Sul: 461 casos. Pesq. Vet. Bras., 27(7):261-268.

SHAHIN, M.; SMITH, B.L.; PRAKASH, A.S. Bracken carcinogenis in the human diet. Mutation Research. 443:69-79, 1999.

SOUTO M.A.M.; KOMMERS, G.D.; BARROS, C.S.L.; PIAZER, J.V.M; RECH, R.R.; RIET-CORREA, F.; SCHILD, A.L. Neoplasias do trato alimentar superior de bovinos associadas ao consumo espontâneo de samambaia (Pteridium aquilinum). Pesq. Vet. Bras. 26:112-122, 2006.

SOUZA, M.V.; GRAÇA, D.L. Intoxicação crônica por Pteridium aquilinum (L) Kuhn (Polypodiaceae) EM BOVINOS. Ciência Rural, [s.I.], v. 23, n. 2, p.203-207, ago. 1993. FapUNIFESP (SciELO). http://dx.doi.org/10.1590/s0103-84781993000200016.

TOKARNIA, C.H.; DÖBEREINER, J.; CANELA, C.F.C. Ocorrência da hematúria enzoótica e de carcinomas epidermóides no trato digestivo superior em bovinos no Brasil. II. Estudos complementares. Pesq. Agropec. Bras. v.4, p. 209-224, 1969.

UENO, H. \& GONÇALVES, P. C. Manual para Diagnóstico das Helmintoses de Ruminantes. JICA, Tokyo, 165 p., 1994. 